\title{
A Three-Component Model of the Control Error in Manual Tracking of Continuous Random Signals
}

\author{
Hans Gerisch, Gerhard Staude, and Werner Wolf, Bundeswehr University \\ Munich, Neubiberg, Germany, and Gerhard Bauch, Hamburg University of \\ Technology, Hamburg, Germany
}

Objective: The performance of human operators acting within closed-loop control systems is investigated in a classic tracking task. The dependence of the control error (tracking error) on the parameters display gain, $k_{\text {display }}$ and input signal frequency bandwidth, $f_{g}$, which alter task difficulty and presumably the control delay, is studied with the aim of functionally specifying it via a model.

Background: The human operator as an element of a cascaded human-machine control system (e.g., car driving or piloting an airplane) codetermines the overall system performance. Control performance of humans in continuous tracking has been described in earlier studies.

Method: Using a handheld joystick, 10 participants tracked continuous random input signals. The parameters $f_{g}$ and $k_{\text {display }}$ were altered between experiments.

Results: Increased task difficulty promoted lengthened control delay and, consequently, increased control error. Tracking performance degraded profoundly with target deflection components above $\mathrm{I} \mathrm{Hz}$, confirming earlier reports.

Conclusion: The control error is composed of a delay-induced component, a demand-based component, and a novel component: a human tracking limit. Accordingly, a new model that allows concepts of the observed control error to be split into these three components is suggested.

Application: To achieve optimal performance in control systems that include a human operator (e.g., vehicles, remote controlled rovers, crane control), (a) tasks should be kept as simple as possible to achieve shortest control delays, and (b) task components requiring higher-frequency $(>\mathrm{I} \mathrm{Hz})$ tracking actions should be avoided or automated by technical systems.

Keywords: tracking performance, human operator, error model

Address correspondence to Hans Gerisch, Bundeswehr University Munich, Institute of Communications Engineering, Werner-Heisenberg-Weg 39, 85577 Neubiberg, Germany; e-mail: hans.gerisch@unibw-muenchen.de.

HUMAN FACTORS

Vol. 55, No. 5, October 2013, pp. 985-1000

DOI: $10.1177 / 0018720813480387$

Copyright $(\mathcal{C}$ 2013, Human Factors and Ergonomics Society.

\section{INTRODUCTION}

Driving a car, operating a construction machine, navigating a ship, or piloting an airplane are all typical cases in which the human operator is an integral component of the overall control system (Craik, 1948; McRuer, Allen, Weir, \& Klein, 1977). Thus, human control contribution is essential for the overall performance and, consequently, an appropriate human-machine interface design represents a key factor for optimal human-machine cooperation. Thus, it is not surprising that human performance in executing control tasks within control systems was and is an intensively investigated topic. It was mainly studied via tracking of a visual target with a hand-operated cursor, as shown in Figure 1a (e.g., Elkind \& Sprague, 1961; Miall, Weir, \& Stein, 1985; Roerdink, Peper, \& Beek, 2005; Van Doorn \& Unema, 2005) as well as in smooth pursuit eye movements (e.g., Voss, McCandliss, Ghajar, \& Suh, 2007; Maioli, Falciati, \& Gianesini, 2007), whereby the eyes have to follow a moving target. Basically, control performance of a human operator and his or her capability of visuomotor adaptation (Reed, Liu, \& Miall, 2003; Engel, Anderson, \& Soechting, 2000) are usually evaluated by analysis of the control error $\Delta x$ (tracking error) and the control delay $\Delta t$ (Figure 1a), both representing time domain parameters.

Another way to describe human tracking behavior is given by frequency domain analysis: The operator is modeled as the controller within a feedback loop, and the controller is characterized by a spectral transfer function. This approach is very simple and effective if only two parameters are used: a general gain $k$ and a constant delay $T$ of the controller. Such a simple concept is used by the so-called McRuer crossover model (McRuer \& Krendel, 1957), originally applied in the design of aircraft control 
systems (Figure 1b). This model allows one to distinguish between various styles of tracking behavior that cannot be discriminated simply by the mean control error $\Delta x$; for example, a large $\Delta x$ might be caused either by fast but oscillatory tracking (underdamped system) or by very sluggish tracking without oscillations — a so-called overdamped system (Jagacinski, 1977; Jagacinski \& Flach, 2003). The basic importance of the delay of a human operator in control tasks was already stressed by Craik (1948) in his basic work: "The first and most marked feature of cerebral process is its time lag or 'central delay.",

For experimental control system analysis, the closed-loop transfer function is usually manipulated by, for example, modifying the feedback branch gain (parameter $k_{\text {displacement }}$ in Figure 1c) while cursor displacement gain (parameter $k_{\text {display }}$ in Figure 1c) in the forward branch remains constant (Buck, 1980; Ferrel, Leifflen, Orliaguet, \& Coello, 2000; Seidler, Bloomberg, $\&$ Stelmach, 2001); as well, the feedback signal can be artificially delayed (Foulkes \& Miall, 2000; Sarlegna, Baud-Bovy, \& Danion, 2010; Stepp, 2009; Wolpert, Miall, Winter, \& Stein, 1992) by inserting $t_{\text {delay }}$ (Figure 1c). An inserted $t_{\text {delay }}$ initially causes an increase of the mean control error; however, as the experiment progresses, the initial decrease in performance is diminished after some adaptation period (Foulkes \& Miall, 2000; Miall et al., 1985).

The gain analysis reveals similar behavior: Despite differences in the control tasks and in the experimental designs, all studies have shown that manual control behavior adapts to system gain parameter $k_{\text {displacement }}$ changes. However, motor performance worsens in "rather lowgain" and "rather high-gain" conditions of the parameter $k_{\text {displacement' }}$ which leads to the generally accepted $U$-shaped relation between gain and control delay (Arnaut \& Greenstein, 1986; Elliot, Lyons, \& Dyson, 1997; Jenkins \& Connor, 1949; Wickens, 1986). Low-gain settings result in large (time-consuming) mechanical amplitudes and, consequently, in extended control delays, whereas high-gain settings require fine (slower) motor skills, resulting in increased control delays and accuracy trade-offs. Thus, distinctly increased difficulty (low-gain and high-gain settings) results in increased control delays, according to Fitts' law (Fitts, 1954).
Another experimental approach to analyzing a closed-loop system (applied, e.g., in the crossover model studies) is to vary the excitation input characteristics while observing the resulting output, which is the control signal $y_{C}(t)$ in Figure 1c (McRuer, Graham, Krendel, \& Reisener, 1965; Rouse \& Gopher, 1977; Sheridan \& Ferrell, 1974). It is known that a tracking task becomes more difficult with increasing dynamics of an unpredictable input signal (as given with an increasing spectral bandwidth of a filtered white noise sequence; Poulton, 1974) and may cause increased human time delay, which can bear the risk of instability. Also, system dynamics were addressed, for example, in visual tracking of slow- and fast-moving discrete targets (Miall, 1996), revealing that the control error was larger for the fast target than for the slow target conditions, and it is increased progressively together with the visual feedback delay (Figure 2 in Miall \& Wolpert, 1996). Furthermore, target tracking was analyzed for different spectral compositions of the input signal (Miall, 1996; Miall et al., 1985; $y_{\mathrm{I}}(t)$ in Figure 1c and Figure $2 \mathrm{a}$ ), and results confirmed the performance decrease with increasing target speed. Certainly, the crossover model inspired an indepth global analysis of tracking behavior mainly focused on stability of the control loop, but these efforts did not further detail different components contributing to the resulting control error.

A step toward greater detail with regard to the control error composition leads to the stage sequence "perception-cognition-action" (the socalled PCA-loop; Gottlieb, 2007), which must be continuously executed during tracking, and each stage within this sequence requires some time for task processing. For a visuomotor task, the retina introduces a delay of 50 to $70 \mathrm{~ms}$, and the motor output component accounts for approximately $70 \mathrm{~ms}$ (composed of the neural transmission of $40 \mathrm{~ms}$ and the muscle electromechanical delay of $30 \mathrm{~ms}$; Cavanagh \& Komi, 1979; Miall \& Wolpert, 1996). Thus, a tracking system itself includes some response delay attributable to causality in sequential processing, which in turn causes a control error, as already discussed (Wickens, 1992, p. 458).

Basic conclusions from all these reports are that (a) there is an upper tracking performance 


\section{(a) continuous tracking}

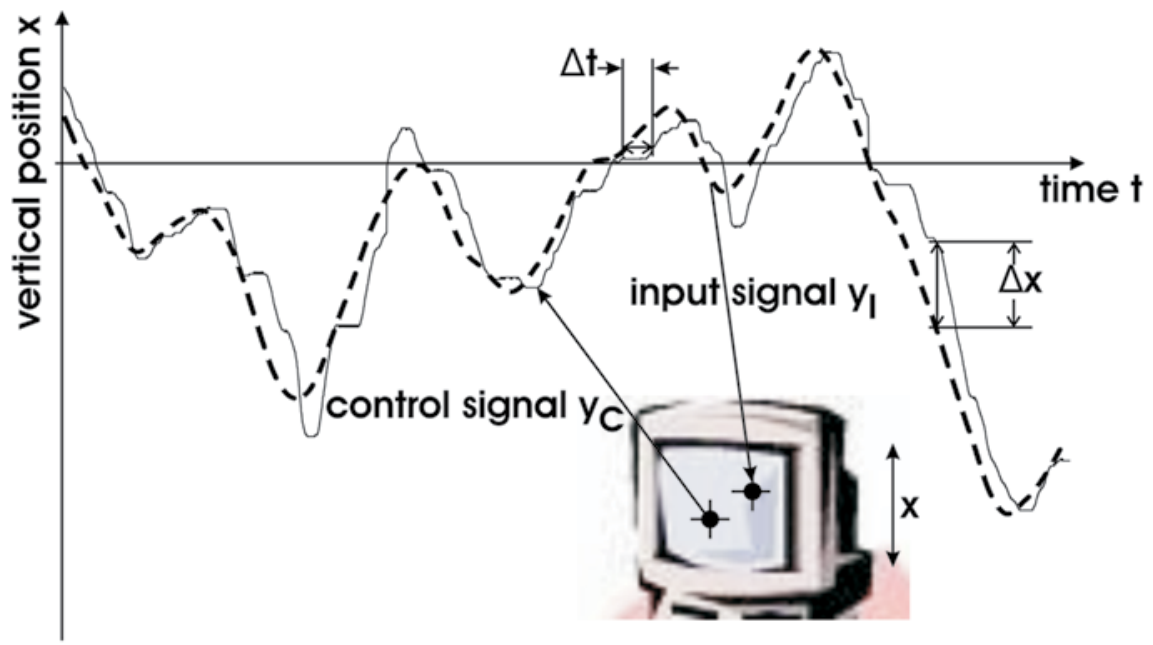

(b) Crossover model

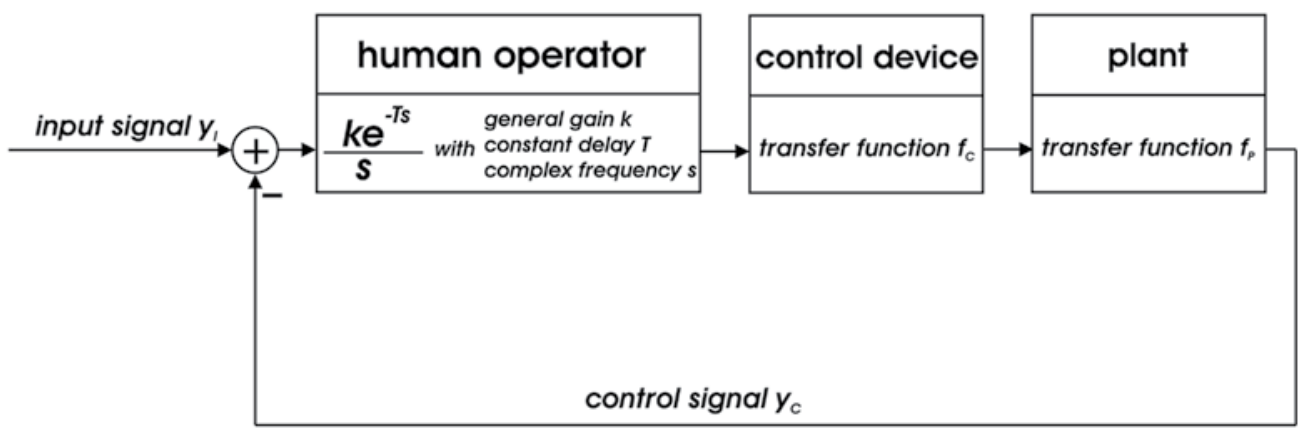

\section{(c) experimental scheme}

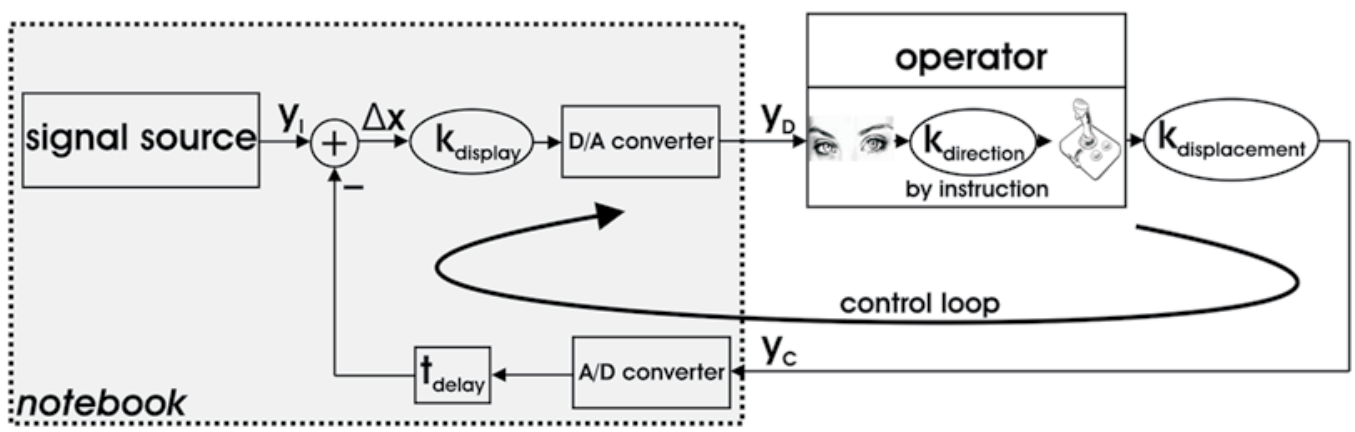

Figure 1. (a) Concept of pursuit tracking experiments. The target cursor (right position on screen) and the tracking cursor (left position) are governed by the input signal $y_{\mathrm{I}}(t)$ and the control signal $y_{\mathrm{C}}(t)$, respectively. The control error $\Delta x$ and control delay $\Delta t$ are standard performance criteria in continuous tracking experiments. (b) The crossover model (McRuer \& Krendel, 1957). In case of a simple tracking feedback device, such as a joystick, $f_{\mathrm{C}}=1$ and $f_{\mathrm{p}}=1$. (c) Experimental scheme of compensatory tracking. The differential signal $y_{\mathrm{D}}(t)$ (also known as "control deviation") controls the target line on the screen. $y_{\mathrm{I}}(t)$ was a band-limited noise signal with cutoff frequency, $f_{g}$. Factors $t_{\text {delay }}$ and $k_{\text {displacement }}$, which were investigated in previous research, were kept constant ( 0 and 1 , respectively) in this study. 
(a)

$$
k_{\text {display }}=4
$$
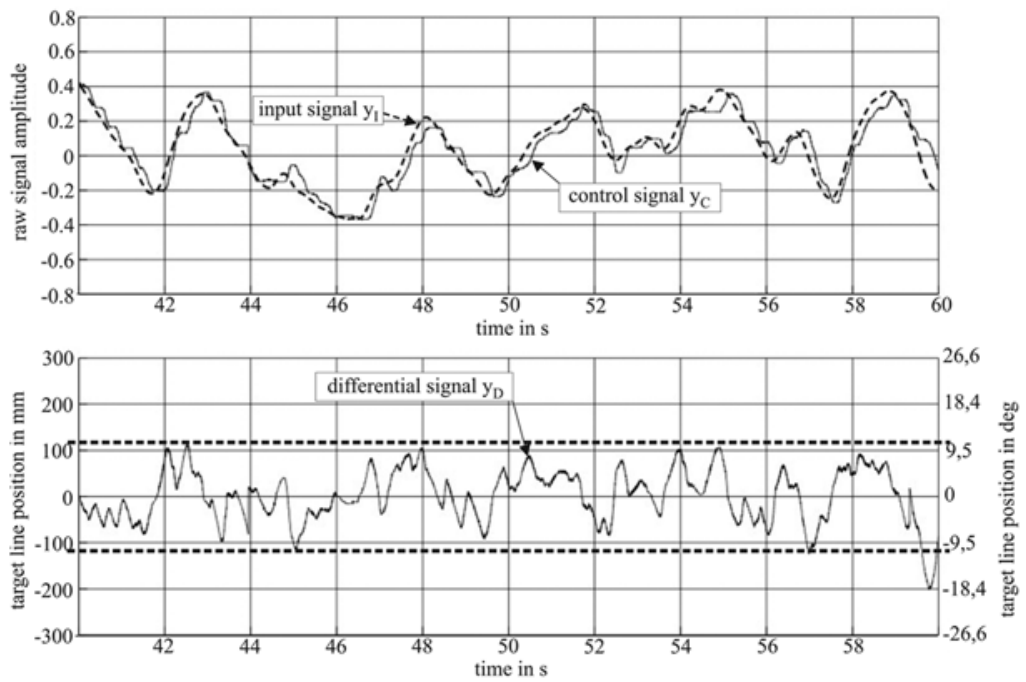

(b)

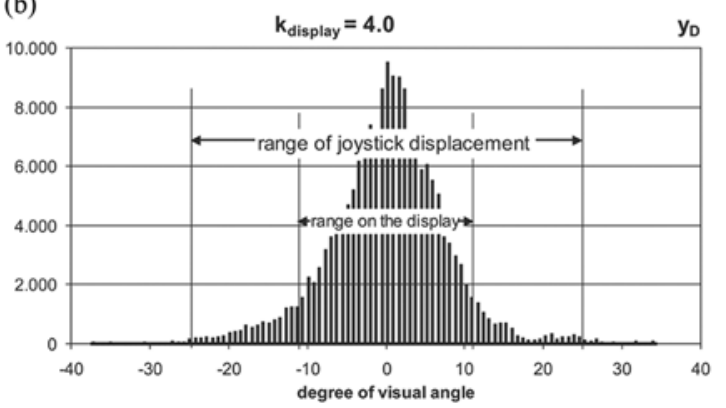

(c)

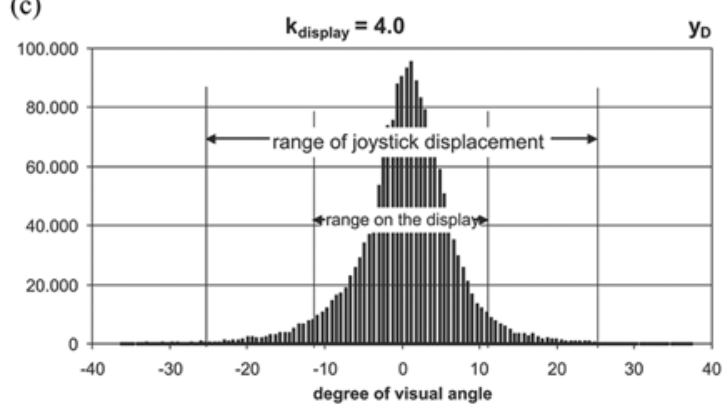

Figure 2. Typical tracking behavior. (a) Segment of original tracking data that show (i) input signal $y_{\mathrm{I}}(t)$ (dashed line in upper plot), (ii) control (output) signal $y_{C}(t)$ (solid line in upper plot), and (iii) differential signal $y_{\mathrm{D}}(t)=$ $k_{\text {display }} \cdot\left[y_{\mathrm{I}}(t)-y_{\mathrm{C}}(t)\right]$ (lower plot). Reasonable tracking performance and a clear (expected) delay between $y_{\mathrm{I}}(t)$ and $y_{C}(t)$ can be recognized. The available range on the display $\left( \pm 11^{\circ}\right)$ is indicated in the lower plot by two horizontal dashed lines, which are rarely exceeded (see text). Factors are $k_{\text {display }}=4, t_{\text {delay }}=0, k_{\text {displacement }}=1, f_{g}=0.4 \mathrm{~Hz}$. P01 represents a typical case. (b) and (c) Amplitude distributions of the differential signal $y_{\mathrm{D}}(t)$ for $k_{\text {display }}^{g}=4$ and $f_{g}=$ 0.4 Hz. (b) Data of a single participant (P01, as in Figure 2a). (c) Pooled data of all 10 participants. The histograms clearly demonstrate the normal distribution of the control error. Also, the similarity of individual and pooled data proves that this tracking behavior is typical for human operators in general.

boundary in the input signal dynamics and (b) the larger the exogenous delay $t$ delay of the visual feedback, the larger the control error $\Delta x$ will be (Foulkes \& Miall, 2000).

The present investigation focuses on specifying the control error sources. Based on the previous reports in literature, the following novel three-component model of control error $\Delta x$ can be hypothesized: The overall control error $\Delta x$ represents the sum of (a) the delay-induced component, (b) the demand-based component for the control processing (which is determined by accuracy trade-offs of the human operator in general), and (c) the human tracking-limit component (i.e., the operator simply does not try to track when the input signal dynamics exceeds an upper frequency $\operatorname{limit} f_{\text {crit }}$ ). This human tracking-limit component is certainly determined by individual factors; however, it is also dependent on the kind of the input signal; $f$ is is constrained by the randomness of the input signal, thus $f_{\text {crit. }}$ will be higher for tracking a single (predictable) sine wave (Poulton, 1974). 
In principle, this error model is compatible with the previously (Etschberger, 1973, 1975) proposed two-component error model suggesting (a) a linear component related to the frequency transfer function of the control loop and (b) a nonlinear component reflecting some internal noise, which proved to be uncorrelated to the input signal $y_{\mathrm{I}}(t)$. (A similar model was supposed by McRuer, 1980.) The linear component of that model can be associated with the control delay $\Delta t$, since a simple delay element can be approximated by a linear transfer function (as done in the crossover model). The uncorrelated nonlinear component suggested by Etschberger (1973, 1975), however, is now divided into the demandbased component and the additional humanboundary component (as mentioned in the previous paragraph), which would be correlated to the input signal; thus, a three-component error model follows. This hypothetical error model for tracking tasks presented in this article was tested with the use of noise input signals of different upper-frequency boundaries $f_{g}$ and different display gains $k_{\text {display }}$.

\section{METHOD}

\section{Participants}

Participants were 10 right-handed healthy men and women ( 5 females, 5 males; between 21 and 31 years of age); they had normal or corrected-to-normal vision and no signs or history of any neurological disorder. Participants, naive of the experimental hypothesis, volunteered to serve in the study and gave their informed consent according to the International Ethical Guidelines for Biomedical Research (Council for International Organizations of Medical Sciences, 2002).

\section{Experimental Setup and Procedure}

Figure 1c shows the basic experimental scheme whereby the participant acts as a human controller within a feedback loop. Participants sat on a chair, adjusted to their individual height. A 14-in. CRT display (HP 1311A, P31 phosphor) providing direct beam control by $y_{\mathrm{D}}$ was positioned at a distance of approximately $60 \mathrm{~cm}$ in front of the participants. The target to track on the screen was a bright horizontal bar $1 \mathrm{~mm}$ thick (i.e., $6^{\prime}$ of visual angle) and $285 \mathrm{~mm}$ long. Zero position was marked on the screen. The bar was vertically deflected (1-D tracking task) and the human operator was instructed to compensate any target line displacement from zero position by an inverse joystick displacement, which follows the rules of a so-called compensatory tracking task. Positive values of $y_{\mathrm{D}}$ correspond to the bar positions above zero position.

The input signal $y_{\mathrm{I}}(t)$ to the control loop (Figure 1c) was constructed from a random signal (white noise) with a Gaussian amplitude distribution, which was band-limited by a thirdorder Butterworth low-pass filter; the cutoff frequency $f$ of the low pass was chosen as $0.2 \mathrm{~Hz}$, $0.4 \mathrm{~Hz}, 0.8 \mathrm{~Hz}$, and $1.2 \mathrm{~Hz}$, respectively. The power of $y_{\mathrm{I}}(t)$ was scaled such that the root mean square value of $y_{\mathrm{I}}(t)$ (magnitude) corresponded to a vertical displacement range of $\pm 5.7^{\circ}$ of visual angle of the bright target bar. Therefore, the root mean square values of the different signals $y_{\mathrm{I}}(t)$ (defined by the different cutoff frequencies) showed the same displacement range on the screen in all cases. The sequences $y_{\mathrm{I}}(t)$ were stored on disk and replayed during the experiment; thus all participants were tested by the same input signal. (Actual maximum amplitudes of the differential signals $y_{\mathrm{D}}(t)$ also depend on the actual control signal amplitudes, and they range up to extremes of $\pm 20^{\circ}$, causing saturation because of the display range of $\pm 11^{\circ}$; these phases, however, cover less than $5 \%$ of the tracking experiment duration, and thus they do not affect the basic results.) Using DIADEM software, we calculated $y_{\mathrm{D}}(t)$ in real time with a sampling rate of $1 \mathrm{kHz}$; it was interfaced to the setup via an I/O card (NI DAQCard ${ }^{\mathrm{TM}}-6024 \mathrm{E}$ ).

The joystick was a two-axial (although only one direction was used), continuously operating, almost frictionless industrial joystick without spring return and damping; that is, a proportional operation was assumed. Joystick displacement range of $\pm 30^{\circ}$ (the positive position range is the direction toward the participant) corresponded to a vertical target displacement range of $\pm 25.8^{\circ}$ visual angle if $k_{\text {displacement }}=1$ and $k_{\text {display }}$ $=1$. (The joystick deflection amplitude $y_{\mathrm{C}}(t)$ required to compensate the actual input signal $y_{\mathrm{I}}(t)$ is independent of the display gain $k_{\text {display }}$.) 
As in previous experiments (Etschberger, 1973, 1975), the differential method of signal tracking (Figure 1c) called compensatory tracking was chosen in contrast to pursuit tracking, depicted in Figure 1a (Strasser \& Platzer, 1972). It provides the advantage of keeping the visual events in the central screen area, avoiding boundary effects and scanning eye movements to a large extent.

Each participant performed 16 trials (each approximately $3 \mathrm{~min}$ in duration) with different input signals $y_{\mathrm{I}}(t)$ and $f_{g}$ and $k_{\text {display }}$ as parameters $\left(k_{\text {display }}\right.$ ranged between 0.25 and 4.0 ), using the dominant hand for joystick control. This set of trials was split into two sessions to prevent fatigue; the sequence of the parameter pairs $\left(k_{\text {display }} / f_{g}\right)$ was randomized to prevent adaptation. Prior to starting the first experimental session, all participants performed as many practice trials as they considered necessary (usually 2 to 3 ) to reduce initial learning effects and to familiarize themselves with the experimental setup.

\section{Data Analysis}

The input signal $y_{\mathrm{I}}(t)$, the control signal $y_{\mathrm{C}}(t)$, and the differential signal $y_{\mathrm{D}}(t)$ (also known as "control deviation") were available as time series for offline data evaluation. Following the rationale of linear control theory (Paul, 2004), the mean square error,

$$
M S E=\frac{\int_{T}\left(y_{I}(t)-y_{C}(t)\right)^{2} d t}{T}
$$

was calculated for the different parameter settings with $T$ as the observation period. In previous works (e.g., Etschberger, 1973), this mean square error (MSE) and the root mean square error $\mathrm{RMSE}=\sqrt{M S E}$ served as appraisal criteria of the feedback control.

The control delay $\Delta t$ between input and control signal was analyzed with the use of correlation techniques. The mean control delay $\bar{\Delta} t$ can be estimated as $\widehat{\Delta} t$ by the so-called lag $\vartheta$ of the maximum of the cross-correlation function $R_{\mathrm{IC}}(\vartheta)$ between input $y_{\mathrm{I}}(t)$ and control signal $y_{\mathrm{C}}(t)$ (Etschberger, 1973, 1975):

$$
R_{I C}(\vartheta)=\frac{1}{T} \int_{T} y_{I}(t) \cdot y_{C}(t+\vartheta) d t
$$

$$
\widehat{\Delta} t=\arg \max _{\vartheta}\left(R_{I C}(\vartheta)\right)
$$

\section{RESULTS \\ General Observations}

Typical raw tracking data are displayed in Figure $2 \mathrm{a}\left(k_{\text {display }}=4.0, f_{g}=0.4 \mathrm{~Hz}\right)$. Obviously, the participant coped with the control task quite well but with some delay. The differential signal $y_{\mathrm{D}}(t)$ typically ranged within $\pm 9^{\circ}$. The power spectrum of the depicted $y_{\mathrm{C}}(t)$ exhibited low-pass characteristics and proved to be mainly restricted to the cutoff frequency $f_{g}$ of the corresponding input signal $y_{\mathrm{I}}(t)$, which also supports the assumption of a basic linear response behavior of the human operator as a first-order approximation.

Figures $2 \mathrm{~b}$ and $2 \mathrm{c}$ elucidate the tracking performance given by the differential signal $y_{\mathrm{D}}=$ $k_{\text {display }} \cdot \Delta x$ more quantitatively. The histogram of Figure $2 \mathrm{~b}$ shows the amplitude distribution of $y_{\mathrm{D}}$ of a single participant; it roughly shows a Gaussian shape, and its 95.4th percentile (which corresponds to two standard deviations in case of a normal distribution of $y_{\mathrm{D}}$ amplitudes) results to $13.4^{\circ}$, which is 4 times the value as determined for $k_{\text {display }}=1.0$. In Figure 2c, however, the actual control errors are pooled for all 10 participants. The similarity of the histogram of a single participant and that of the results pooled across all participants demonstrates the homogeneity of the data within the group.

\section{Time Lag (Control Delay)}

The most striking observation, shown in Figure $2 \mathrm{a}$, is the time lag $\Delta t$ of $y_{\mathrm{C}}(t)$ with respect to $y_{\mathrm{I}}(t)$, which is intrinsic to human operators. In principle, the perception-to-action delay $\Delta t$ of humans can be compensated in the diagram if the delay $\Delta t$ is known. $\widehat{\Delta} t$ can be estimated according to Equation (3) to construct the "delay-compensated" differential signal $\bar{y}_{D}(t)=k_{\text {display }} \cdot\left[y_{\mathrm{I}}(t)-y_{\mathrm{C}}(t+\right.$ $\widehat{\Delta} t)]$, which clearly shows an amplitude reduction, compared to $y_{\mathrm{D}}(t)$ in Figure $2 \mathrm{a}$. Thus, the time lag is confirmed as an essential factor in tracking performance.

More comprehensively, Figure $3 \mathrm{a}$ depicts the estimated time lag $\widehat{\Delta} t$ between input $y_{\mathrm{I}}(t)$ and control signal $y_{\mathrm{C}}(t)$, now for all parameter 


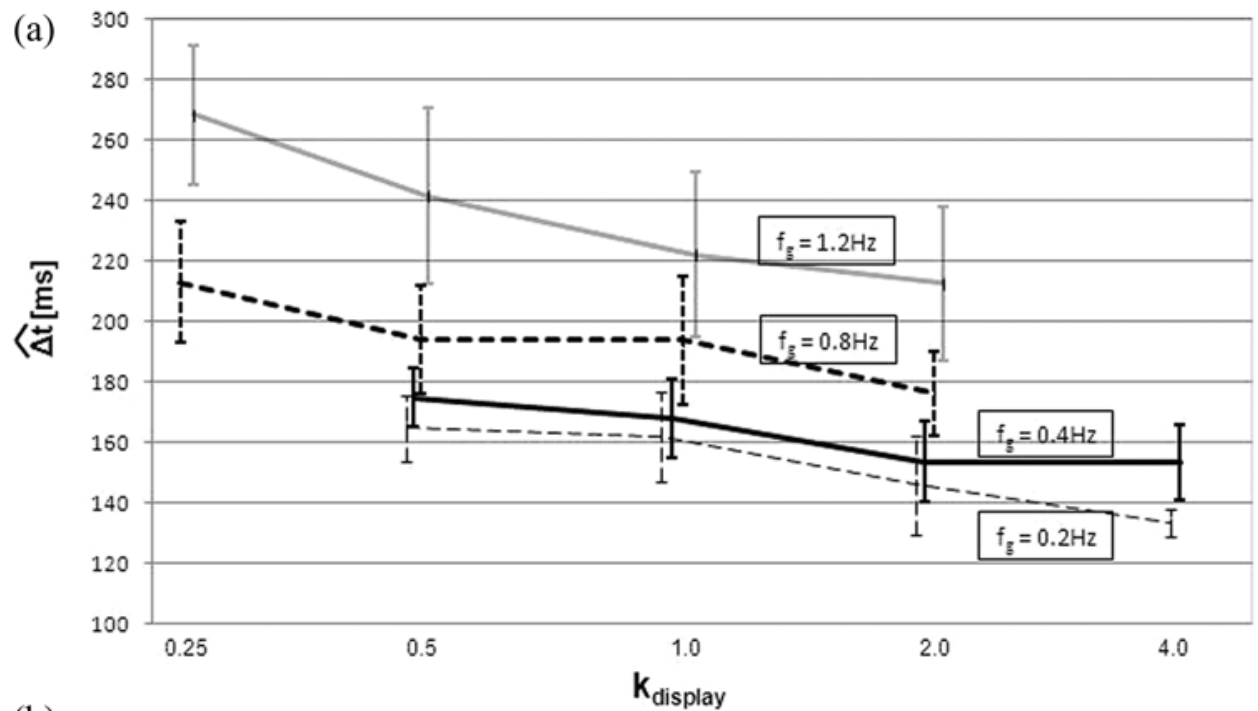

(b)

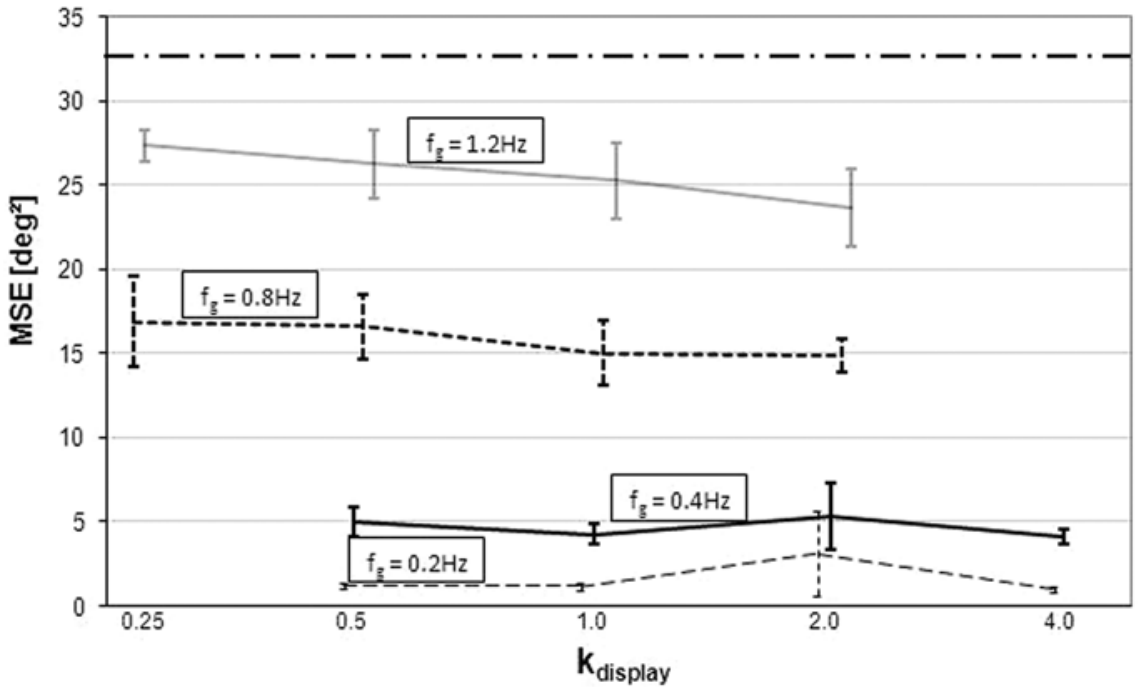

Figure 3. Observed control parameters. (a) Estimated time lag $\widehat{\Delta} t$ between input signal $y_{\mathrm{I}}(t)$ and control signal $y_{\mathrm{C}}(t)$ with cutoff frequency $f_{g}$ as parameter. (b) Mean square error (MSE) as well as root mean square error (RMSE). The tracking performance is almost independent on the display gain $k_{\text {display }}$; however, $f_{g}$ shows a significant influence. The open-loop condition $\left[y_{\mathrm{C}}(t)=0\right]$ leads to the theoretical upper boundary of mean square error indicated in (b) by the dash-dotted line. Data presented in both diagrams are pooled for all participants, and the graphs are slightly horizontally shifted to avoid overlapping of the vertical bars indicating the standard deviations.

combinations of the display gain and cutoff frequencies. Generally, it can be observed that for increasing $k_{\text {display, }}$ the time lag $\widehat{\Delta} t$ slightly decreases, but there is a distinct rise of $\widehat{\Delta} t$ for increasing $f_{g}$ of the input signal $y_{\mathrm{I}}(t)$. Thus, $\widehat{\Delta} t$ does indeed depend on the dynamics of $y_{\mathrm{I}}(t)$ : An increase of $k_{\text {display }}$ directly leads to a rise in target speed, but an increase of $f_{g}$ causes higher speed as well as a higher "complexity" of the target movement. 


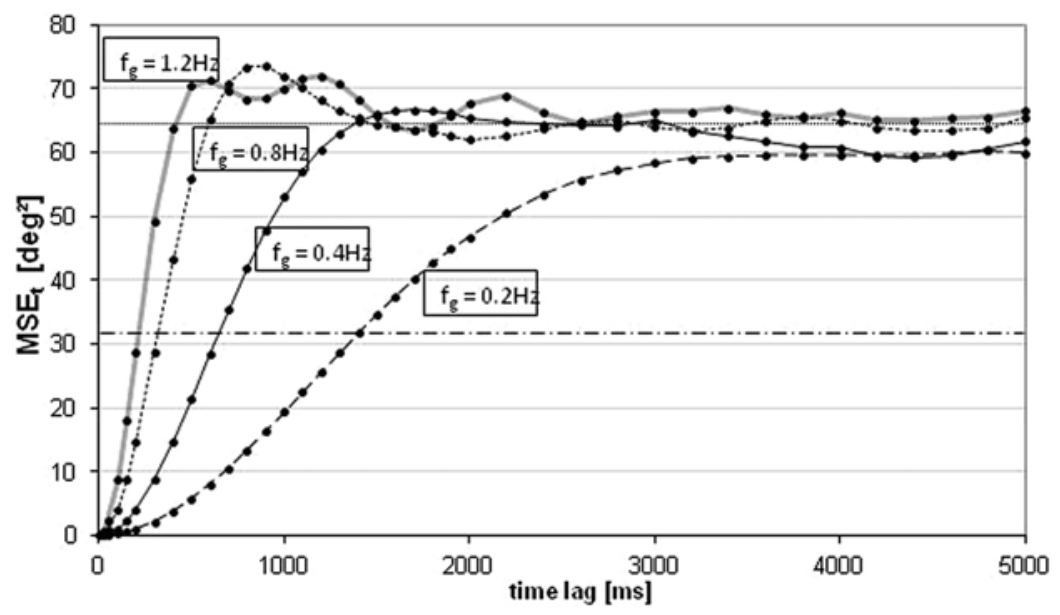

Figure 4. $M S E_{t}$ obtained for an ideal but delayed human operator with $y_{C}(t)=$ $y_{\mathrm{I}}[(t-\Delta t)]$ as a function of the time lag (control delay) $\Delta t$ with $f_{g}$ as parameter. Clearly, the $M S E_{t}$ is increasing systematically with increasing $\Delta t$ and is converging to $\approx 65\left({ }^{\circ}\right)^{2}$ (indicated by the dotted line) as the sum of $M S\left[y_{\mathrm{I}}(t)\right]$ and $M S\left[y_{\mathrm{C}}(t)=y_{\mathrm{I}}(t-\Delta t)\right]$ for large $\Delta t$. The mean square of the input signal $y_{\mathrm{I}}(t), M S\left(y_{\mathrm{I}}\right)=32.49\left(^{\circ}\right)^{2}$, resulting from the open-loop condition $\left[y_{\mathrm{C}}(t)=0\right]$, is indicated by the dash-dotted line.

\section{Mean Square Error and RMSE}

Finally, the mean square error between $y_{\mathrm{I}}$ and $y_{\mathrm{C}}$ according to Equation (1) and the RMSE are shown as a function of the display gain with $f_{g}$ as a parameter in Figure $3 \mathrm{~b}$ (see on the left ordinate the mean square error scaling and on the right ordinate the RMSE scaling). Additionally, the standard deviations are indicated. The RMSE values for $f_{g}=1.2 \mathrm{~Hz}$ tend toward the root mean square value of $y_{\mathrm{I}}(t)$ (i.e., no feedback signal from joystick): Root mean square of $y_{1}=$ $5.7^{\circ}$; correspondingly, $M S\left(y_{\mathrm{I}}\right)=32.49\left(^{\circ}\right)^{2}$. This finding indicates some upper boundary of the participants' performance, since their tracking does not really seem to correspond to the target movement.

Figure 4 illustrates that at least some part of the mean square error can be expected from the intrinsic time delay $\Delta t$ characterizing human operators. We determined this component, $M S E$, by simulating an ideal human operator who introduces only a human time lag $\Delta t$, formally described by $y_{\mathrm{C}}(t)=y_{\mathrm{I}}(t-\Delta t)$, but no other control deviation (Figure 4). MSE increases with increasing $\Delta t$ as expected on the basis of theoretical considerations $\left(M S E_{t}\right.$ profile with respect to $\Delta t$ can be predicted from the transfer function of the low-pass filter (third-order Butterworth) used to restrict the frequency bandwidth of $y_{\mathrm{I}}[t]$ ). For large $\Delta t$, the $M S E_{t}$ profile converges to the sum of the mean square of $y_{\mathrm{I}}(t)$ and $y_{\mathrm{C}}(t)=y_{\mathrm{I}}$ $(t-\Delta t)$ (Unbehauen, 2008):

$$
\begin{gathered}
M S\left[y_{I}(\mathrm{t})+y_{I}(t-\Delta t)\right]=M S\left[y_{I}(t)\right]+M S\left[y_{I}(t\right. \\
-\Delta t)]=2 M S\left[y_{I}(t)\right] .
\end{gathered}
$$

Thus, if part of the raw mean square error shown in Figure $3 \mathrm{~b}$ is specifically attributed to the control delay $\Delta t$, the raw mean square error values can be split into a time lag component $M S E_{t}$ and a component $M S E_{\text {op }}$ caused by the operator's perception-to-action transfer function, thus resulting in the following model:

or

$$
M S E=M S E_{\mathrm{op}}+M S E_{t}
$$

$$
M S E_{\mathrm{op}}=M S E-M S E_{i} .
$$

Figure 5a depicts this estimated $M S E_{\text {op }}$ (with same data as in Figure 3b) as a function of the display gain with $f_{g}$ as parameter. Obviously, the residual operator-induced component $M S E_{\text {op }}$ 
(a)

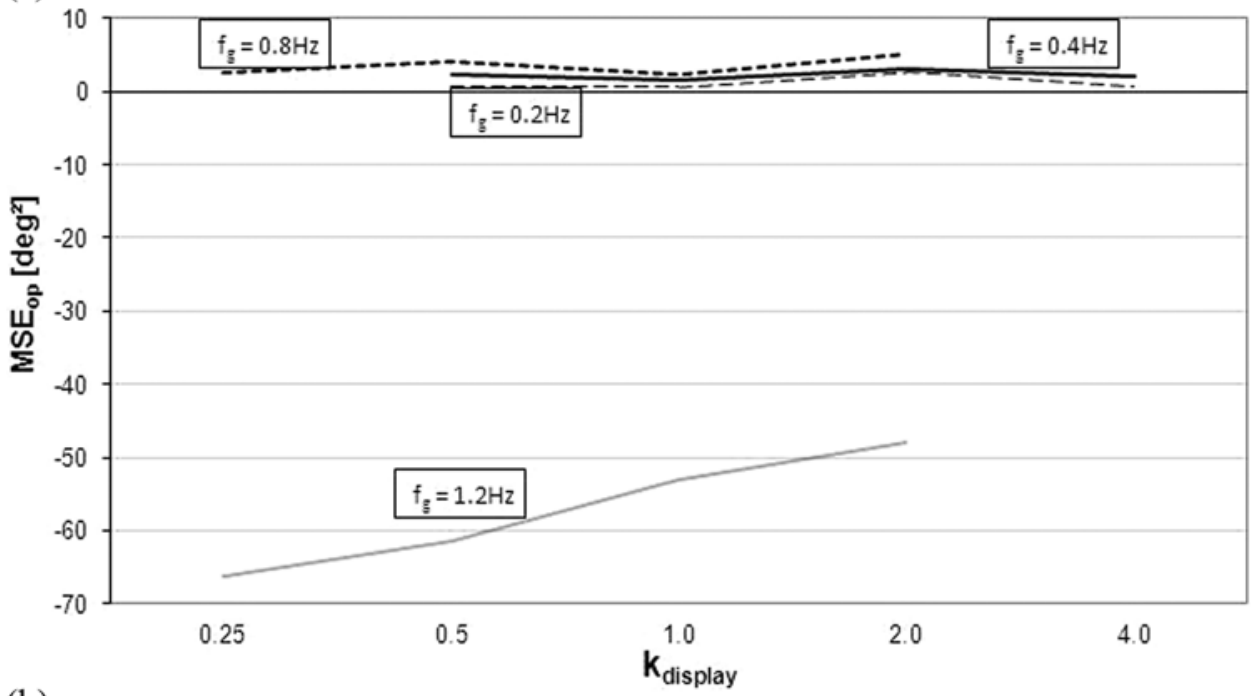

(b)

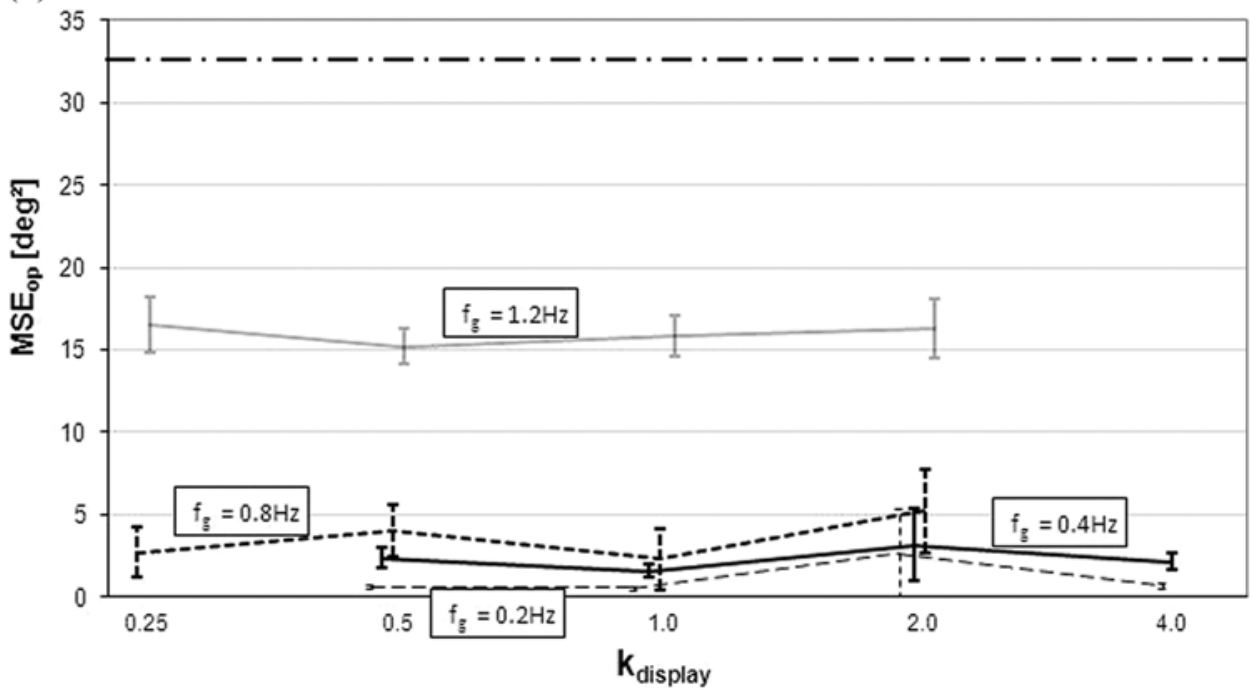

Figure 5. (a) Estimated operator-specific $M S E_{\text {op }}$ achieved by subtracting the expected $M S E_{t}$ contribution attributable to simple time lag (as shown by Figure 4) from the measured mean square error values (Figure $3 b$ ). $M S E_{\text {op }}$ converges to values between $1\left(^{\circ}\right)^{2}$ and $5\left(^{\circ}\right)^{2}$ except for the condition $f_{g}=1.2 \mathrm{~Hz}$. In the latter case, the large (mathematically impossible) negative values indicate that this two-component control error splitting model is not further valid for $f_{g}$ $>0.8 \mathrm{~Hz}$. (b) Same as Figure 5a, but now $M S E_{\text {op }}\left(f_{g}=1.2 \mathrm{~Hz}\right)$ is calculated with the suggested three-component error model. Again, the graphs are horizontally shifted to avoid overlapping of the vertical bars indicating the standard deviation.

ranges somewhere between $1\left(^{\circ}\right)^{2}$ and $5\left(^{\circ}\right)^{2}$, which means that the basic control behavior of the human operator is independent of the specific experimental conditions, as long as the task complexity (in our study, given by $f_{g}$ ) does not exceed the operator's performance limit. Obviously, this "overload" occurs at some initial frequency $0.8 \mathrm{~Hz} \leq f_{\text {crit }}<1.2 \mathrm{~Hz}$. Therefore, the operator's tracking behavior will not match the estimation procedure of Equation (6) for the 
condition $f_{g}=1.2 \mathrm{~Hz}$, which is clearly indicated by the negative values for a squared measure, such as $M S E_{\text {op }}$ in Figure 5a. This observation supports the assumption that frequency components $f>f_{\text {crit }}$ of the input signal were not included in the perception-action response of the operator.

Based on this assumption, an extended threecomponent error model was suggested in the Introduction: First, $M S E_{\text {op }}$ is composed of two components: (a) resulting from the tracking signal components of the lower-frequency band $(f<$ $f_{\text {crit }}$ ) and (b) resulting from the tracking signal components of the upper-frequency band $(f \geq$ $\left.f_{\text {crit }}\right)$. For cutoff frequencies $f_{g} \geq f_{\text {crit }}$, this results in the following:

$$
\begin{gathered}
M S E_{\text {op }}\left(f_{g}\right)=c_{1}^{*} M S E_{\text {op }}\left(f_{g} \leq f_{\text {crit }}\right)+c_{2}^{*} M S E_{\text {op }}\left(f_{\text {crit }}\right. \\
<f \leq f), \\
\text { with } \mathrm{c}_{1}=\frac{f_{\text {crit }}}{f_{g}} \text { and } \mathrm{c}_{2}=1-\mathrm{c}_{1} .
\end{gathered}
$$

For $f_{g}=1.2 \mathrm{~Hz}$ and assuming that $f_{\text {crit }}=0.8$ $\mathrm{Hz}$, this results in

$$
\begin{aligned}
& M S E_{o p}\left(f_{g}=1.2 \mathrm{~Hz}\right)=\frac{2}{3} * M S E_{o p}(f \leq 0.8 \mathrm{~Hz}) \\
& +\frac{1}{3} * M S E(0.8 \mathrm{~Hz}<f \leq 1.2 \mathrm{~Hz})
\end{aligned}
$$

The factors $c_{1}=2 / 3$ and $c_{2}=1 / 3$ of the two terms scale the two components according to their bandwidth within $y_{\mathrm{I}}(t)$ (i.e., $0.0-0.8 \mathrm{~Hz}$ and $0.8-$ $1.2 \mathrm{~Hz}$, respectively, assuming a uniform energy distribution over frequency). The result is depicted in Figure 5b: The curves obtained for the cutoff frequencies $f_{g}=0.2,0.4$, and $0.8 \mathrm{~Hz}$ correspond to the curves in Figure 5a. The curve for the cutoff frequency $f=1.2 \mathrm{~Hz}$ is changed, revealing a value around $16\left({ }^{g}\right)^{2}$, which is now reasonable in contrast to the theoretically impossible negative value of $M S E_{\text {op }}\left(f_{g}=1.2 \mathrm{~Hz}\right)$ obtained through simple control error splitting, according to Equation (6) and presented in Figure 5a.

\section{DISCUSSION}

The data reported here fit to previous findings on human tracking behavior; in particular, the results reaffirm that the transfer function of the human tracking control loop is limited to approximately $1 \mathrm{~Hz}$ (Etschberger, 1975; Knight, 1987), which is also in line with the reported Nyquist frequency $f$ of around $1 \mathrm{~Hz}$ based on the crossover model (McRuer, 1995). First and foremost, however, the data lend support to the threecomponent error model presented here, namely, that the control error of a human operator can be divided into (a) a delay-induced component, (b) a demand-based component for the control processing (which is determined by accuracy trade-offs of the human operator in general), and (c) a human tracking-limit component.

\section{Display Gain and Time Lag}

In the investigated range of display gain, small display gains $k_{\text {display }}$ required a longer control system acting time indicated by a longer time lag (Figure 3a). Small display gains lead to small and less distinctive target movements - thus impeding visual perception and, in turn, increasing the visuomotor coordination time, since the visuomotor system supervises the performed tracking (Holst \& Mittelstaedt, 1950). Another reason for the larger time lag for smaller display gains can also be the so-called error dead zone (Wolpert et al., 1992). This term indicates a critical displacement threshold that the control deviation must exceed before the human operator will perform correcting movements to compensate for the deviation (Joiner \& Shelhamer, 2009; Reed et al., 2003). It can be speculated that this dead zone size corresponds to the residual tracking $M S E_{\text {op }}$ shown in Figure $5 b$.

\section{Further Support for the Three- Component Model of the Control Error (Tracking Error)}

The mean square error (and RSME) of the control error $\Delta x$ (shown in Figure $3 b$ ) characterizes human tracking performance. As previously reported (Etschberger, 1973), the flat, almost horizontal mean square error curves (Figure 3b) indicate that human tracking performance is almost independent of various $k_{\text {display }}$ values; as the required manual movement is also independent of $k_{\text {display }}$ because of constant $k_{\text {displacement }}$, only the perceptual process differs since changed display gains result in different target movement velocities. However, the target movement velocity range used is beyond any perceptual limits because of the high contrast 

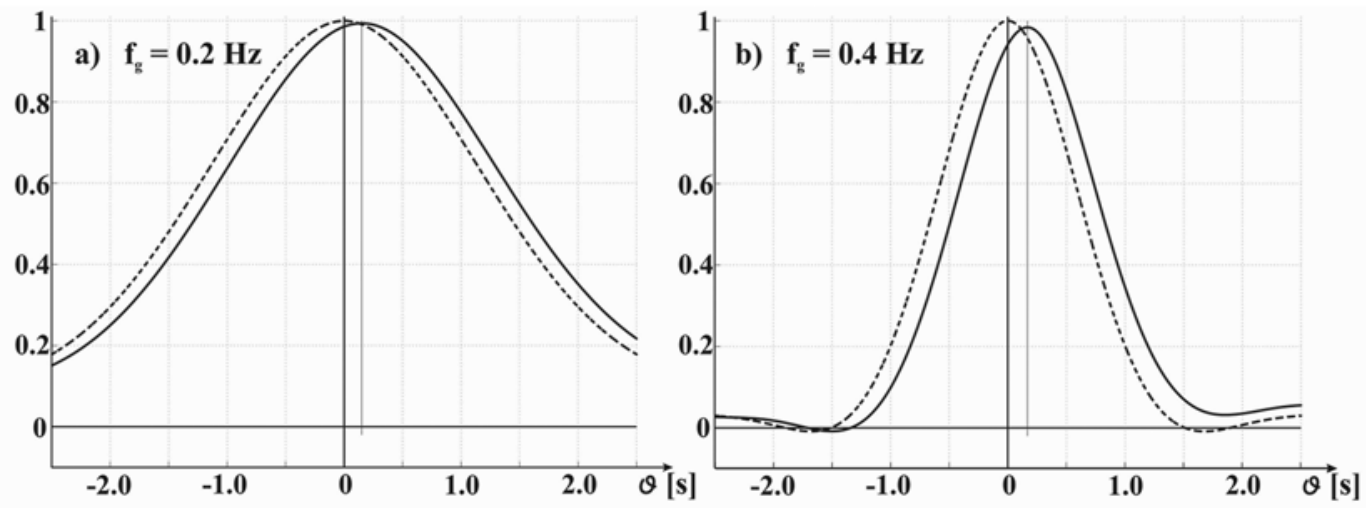

$\cdots \cdot-\cdot R_{I I}\left(\right.$ autocorrelation function of $\left.y_{1}(t)\right)-R_{I C}$ (cross-correlation function between $y_{1}(t)$ and $y_{c}(t)$ )
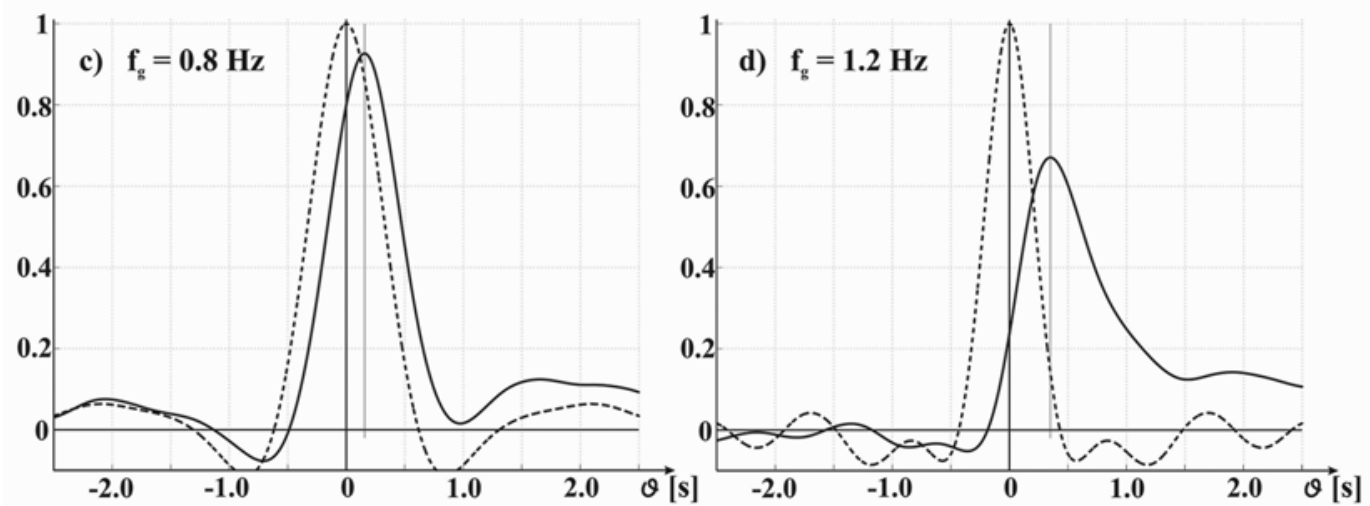

Figure 6. Typical correlation functions of $y_{\mathrm{I}}(t)$ and $y_{\mathrm{C}}(t)$ for the different values of $f_{g}$. The dotted line shows the autocorrelation function $R_{\mathrm{II}}(\vartheta)$ of the input signal $y_{\mathrm{I}}(t)$. The solid line represents the crosscorrelation function $R_{\mathrm{IC}}(\vartheta)$ between input signal $y_{\mathrm{I}}(t)$ and control (output) signal $y_{\mathrm{C}}(t)$. Their correlation strength drastically drops at $f_{g}=1.2 \mathrm{~Hz}$, indicating the operator's tracking limit. (Participant P01)

target (Burr \& Ross, 1982; Campbell \& Maffei, 1981). Hence, the control error (Figure 3b) as well as the control delay (Figure 3a) strongly depends on the input signal bandwidth $f$, which reflects the characteristic behavior of the total control loop (i.e., of both perception and action) engaged in the tracking task and confirms previous reports (e.g., Wickens, 1986).

When the observed score of the mean control error reached the score obtained for $y_{\mathrm{C}}=0$ (i.e., no joystick feedback), the performance limit appears to have been reached as well. In fact, participants tended to $M S E \approx 27\left(\left(^{\circ}\right)^{2}\right.$ and RMSE $\approx 5.3^{\circ}$ for $f_{g}=1.2 \mathrm{~Hz}$, whereas $M S E=32.49\left(^{\circ}\right)^{2}$ and $\mathrm{RMSE}^{g}=5.7^{\circ}$ are obtained for $y_{\mathrm{C}}=0$ (see Figure $3 \mathrm{~b}$ ). Such a dissociation of the control signal $y_{C}(t)$ from the input signal $y_{\mathrm{r}}(t)$ can be further checked with the use of correlation techniques as an additional analysis tool. As long as the control signal $y_{\mathrm{C}}(t)$ reflects the input signal $y_{\mathrm{I}}(t)$ (as it is expected in a control loop), the shape of the cross-correlation between $y_{\mathrm{I}}(t)$ and $y_{\mathrm{C}}(t)=$ $f\left[y_{\mathrm{I}}(t)\right]$, named $R_{\mathrm{IC}}(\vartheta)$ according to Equation (2), should also somehow reproduce the autocorrelation of $y_{\mathrm{I}}(t)$, named $R_{\mathrm{II}}(\vartheta)$, which is identical with the cross-correlation between $y_{\mathrm{I}}(t)$ and $y_{\mathrm{I}}(t)$. Both correlation functions are shown in Figures $6 \mathrm{a}$ through $6 \mathrm{~d}$ for the four different cutoff frequencies $f_{g}$.

$R_{\text {IC }}(\vartheta)$ (solid lines) and $R_{\text {II }}(\vartheta)$ (dotted lines) coincide in shape for $f_{g}=0.2,0.4$, and $0.8 \mathrm{~Hz}$, and as expected, the widths of the peaks of the correlation functions directly correspond to the different cutoff frequencies $f_{g}$. Furthermore, $R_{\mathrm{IC}}(\vartheta)$ shows a lag as a consequence to the operator response delay. But the coupling strength 


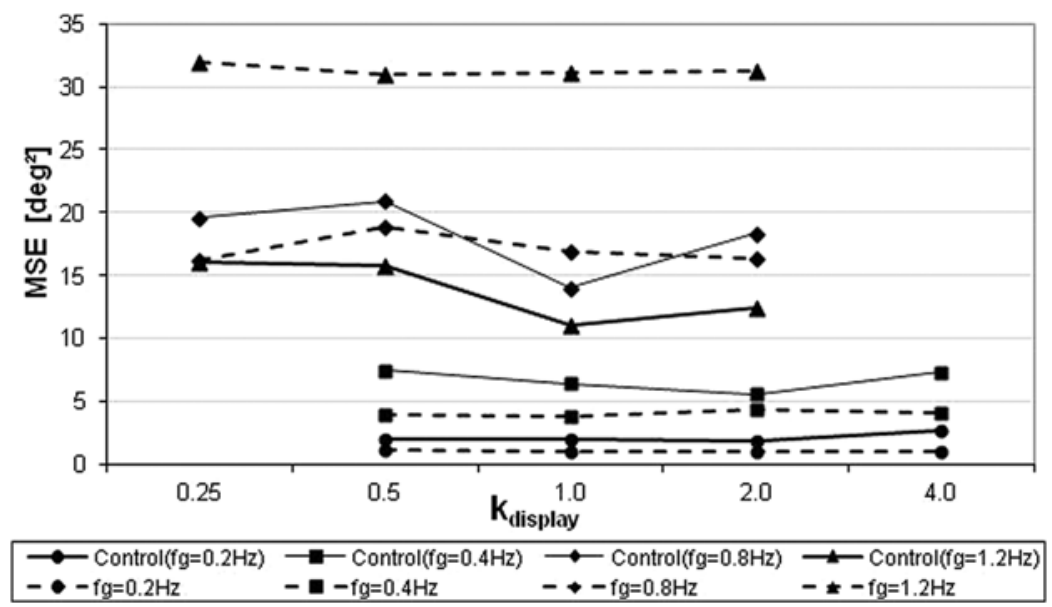

Figure 7. Mean square error obtained in the "normal" experiment (dashed lines) together with the mean square error obtained in the pilot experiment using the control signal of a previous trial of same participant as input signal $y_{\mathrm{I}}(t)=$ $y_{\text {Creused }}(t)$ (solid lines). Graphs are depicted as a function of $k_{\text {display }}$ with $f_{g}$ as parameter. Clearly, the curves for both conditions at $f_{g}=1.2 \mathrm{~Hz}$ deviate from each other, whereas those at the other frequencies do not. (Participant P01)

expressed by the maximum amplitude of $R_{\mathrm{IC}}(\vartheta)$ significantly drops from $\sim 0.95$ to $\sim 0.65$ at $f_{g}=$ $1.2 \mathrm{~Hz}$. Thus, an upper boundary of human tracking performance can be placed at a cutoff frequency $f_{\text {crit }}$ somewhere between $0.8 \mathrm{~Hz}<f_{g}<$ $1.2 \mathrm{~Hz}$. For $f_{g}=1.2 \mathrm{~Hz}$, however, the shape of $R_{\mathrm{IC}}(\vartheta)$ looks quite different from the shape of $R_{\mathrm{II}}(\vartheta)$, which emphasizes that some parts of the input signal $y_{\mathrm{I}}(t)$ were not included in the response process.

This aspect is further supported by a pilot experiment that involved the following experimental technique: If some higher-frequency components of the input signal $y_{\mathrm{I}}(t)$ are not further represented in the response $y_{\mathrm{C}}(t)$ (i.e., the human operator acts as a filter), then such low tracking performance attributable to higherfrequency components should not be observable when reusing such a recorded control signal $y_{\mathrm{C}}(t)$ in a subsequent experiment as input signal $y_{\mathrm{I}}(t)$. Accordingly, the pilot experiments used the recorded control signals $y_{C}(t)$ as input signal $y_{\mathrm{I}}(t)=y_{\text {Creused }}(t)$. Figure 7 depicts the resulting mean square error curves with solid lines in comparison to the data from the original experiments represented by the dashed lines.

Both curves for the same cutoff frequency basically coincide; however, for $f_{g}=1.2 \mathrm{~Hz}$, they differ: The solid line rather matches the mean square error curve for $f_{a}=0.8 \mathrm{~Hz}$ instead of the original curve for $f_{g}=1.2 \mathrm{~Hz}$. Again, the correlation analysis was performed; results are displayed in Figure 8.

Comparing Figures 6 and 8, the $R_{\mathrm{IC}}(\vartheta)$ obtained for the cutoff frequencies $f_{g}=0.2,0.4$, and $0.8 \mathrm{~Hz}$ (Figures 8a through 8c) coincide with the corresponding curves of Figures 6a through 6c. Remarkably in Figure 8d, the curve for $f_{g}=1.2 \mathrm{~Hz}$ obtained with the recorded input signal $y_{\mathrm{I}}(t)=$ $y_{\text {Creused }}(t)$ is changed with respect to Figure $6 \mathrm{~d}$ : It is now rather similar to the case of $f_{g}=0.8 \mathrm{~Hz}$ (Figure $8 \mathrm{c}$ ). This observation again strongly supports the assumption that frequency components $f>0.8$ $\mathrm{Hz}$ of the input signal were not included in the perception-action response of the operator. It also affirms the existence of a human tracking-limit component of the overall control error $\Delta x$.

\section{A Flash-Like Epilogue on Conceivable Applications}

Better understanding of human tracking behavior as well as its limitations can support the optimizing of human-machine interface designs. The knowledge of the upper performance limit is important when a human operator should control a system whose natural 

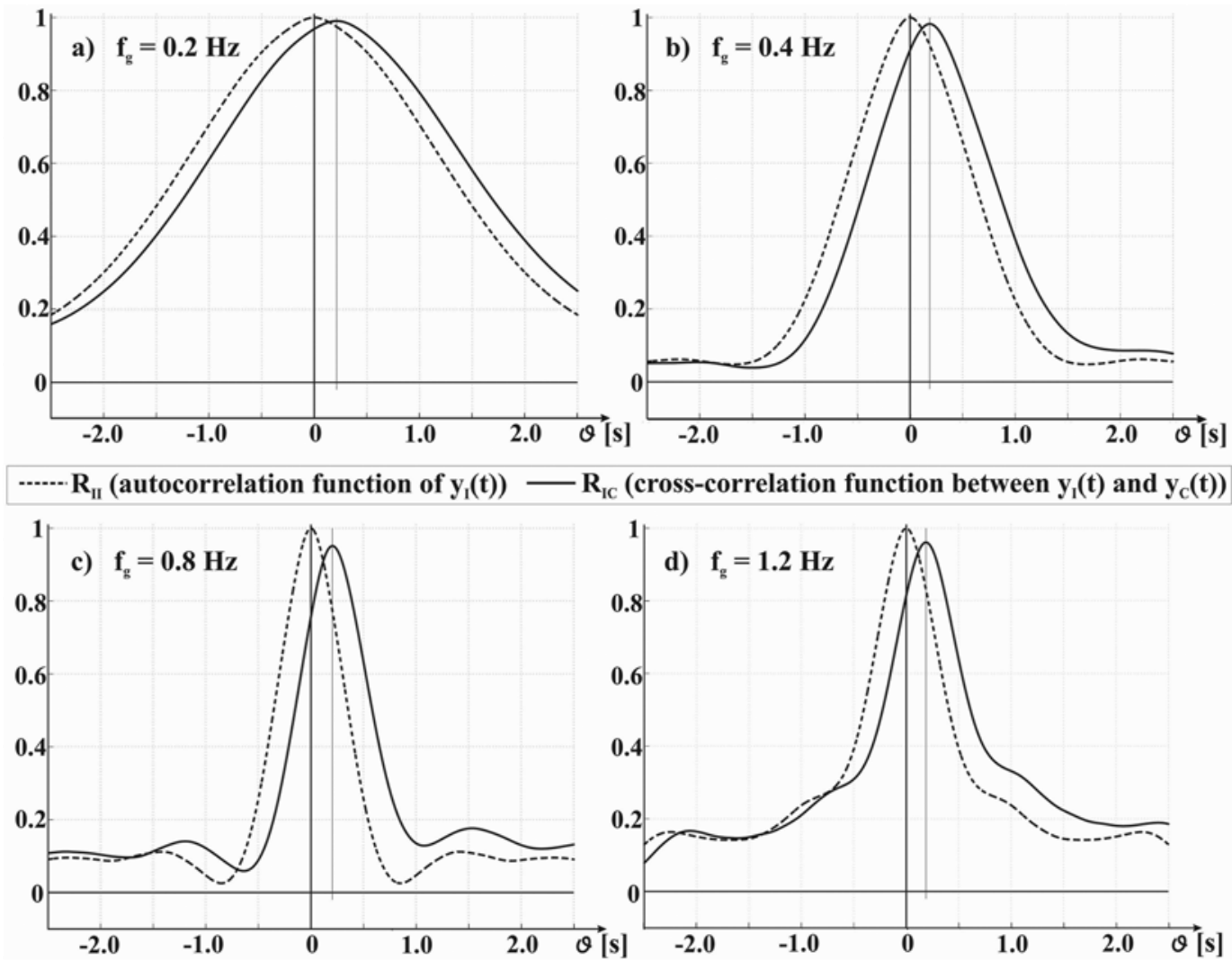

Figure 8. Same as Figure 6 but now for the modified condition $y_{\mathrm{I}}(t)=y_{\text {Creused }}(t)$. Clearly, only Panel (d) for $f_{g}=1.2 \mathrm{~Hz}$ is different from that of Figure $6 \mathrm{~d}$, but it is very similar to Panel (c), indicating that the input signals are also of similar characteristic.

frequency exceeds $f_{\text {crit }}$ of the operator and thus the operator will not be able to perform the job successfully. The worst case would be when this limitation leads to instability of the system; to avoid this case was the original motivation for the investigations of McRuer (McRuer \& Krendel, 1957). A direct application of such a lab tracking experiment (as described in this article) can be found in remote-controlled vehicles, such as robotic rovers or unmanned aerial vehicles. The most popular tracking task, however, is the steering of a vehicle like an aircraft or a car; nevertheless, there are also other tracking situations, such as controlling the load of a crane when moving it around obstacles, as described in a very recent report (Potter \& Singhose, 2013). In all those cases, the controlled system can enter an oscillating state whereby the oscillating frequency can exceed $f_{\text {crit }}$. It was shown that an input shaping of the operator's command signal to the plant leads to suppression of those oscillations and to improved tracking performance (Khalid, Huey, Singhose, Lawrence, \& Frakes, 2006); this input shaping suppresses higher-frequency components not supporting the control, which basically links to our result of the third error component.

\section{CONCLUSION}

As early as 1947, Craik described interference between control error and response time: "the periodic . . . nature of the time-record of tracking errors, showing a spectrum with a predominant frequency of about $0.5 \mathrm{sec}$ with a smaller cluster of frequencies from 0.25 to 1 sec" (Craik, 1947, p. 56). The present study revives this theory and suggests 
splitting the control error into a delay-induced component, $M S E$, and a residual operatorinduced component, $M S E_{\mathrm{op}}$. Obviously, $M S E_{\text {o }}$ seems to be constant as long as the complexity of control does not exceed a certain limit indicated by $f_{\text {crit }}$. Beyond this performance, a further splitting of the residual operator-induced component, $M S E_{\text {op }}$, into a demand-based component for the control processing (=MSE for input signals of a bandwidth smaller than $f_{\text {crit }}$ ) and a human tracking-limit component $(=0$ for input signals of a bandwidth smaller than $f_{\text {crit }}$ ) is required.

This result leads to the assumption that the unpredictable signals effectively tracked by human operators are of a bandwidth that is less than $f_{\text {crit }}$ when $0.8 \mathrm{~Hz}<f_{\text {crit }} \leq 1.2 \mathrm{~Hz}$. The questions of whether this limitation can generally be shifted through training or whether predictable signals (e.g., while steering a car along a wide curve) can be better tracked given reduced control delays are left open as further topics of upcoming research. Another important question for application is whether "overload information" (i.e., spectrum $>0.8 \mathrm{~Hz}$ ) leads per se to a longer control delay $\Delta t$ because of the additional cognitive load and, consequently, to reduced tracking performance; if so, the information presented to the operator should be restricted to the useful frequency range. Our data, in line with the reports on input shaping (Potter \& Singhose, 2013), show some indication of this finding (Figure 3a); however, final conclusions require further experimental efforts. In particular, the inclusion of typical plant transfer functions in this research can bridge lab results and their applications.

\section{ACKNOWLEDGMENTS}

Many thanks are due to the anonymous reviewers who inspired the revision of this article with some very sophisticated ideas. The authors also are indebted to W. Weber and J. Dochtermann for their support in the realization of the study. The support of R. Noragon and D. Mook as native speakers was very helpful, as were the critical comments of M. Sharikadze to the original draft. This work was supported by DFG Grant Wo 364/7-3 to W. Wolf and G. Staude and by DFG Excellence-Cluster "Cognition for Technical Systems" Project CogMan to W. Wolf.

\section{KEY POINTS}

- Human tracking performance is dependent on the target movement dynamics but almost independent of the display gain factor.

- The known interference between the control error and the control delay is reinvestigated.

- A novel model for the control error (tracking error) is presented that comprises (a) a delay-induced component, (b) a demand-based component, and (c) a human tracking-limit component.

- There is an upper tracking performance boundary: The transfer function of the human tracking control loop is limited at around $1 \mathrm{~Hz}$.

- Authors of future studies should investigate the influence of training on this limitation, that is, whether possible reduction of control delay, and, subsequently, of control error helps humans in dealing with predictable signals.

\section{REFERENCES}

Arnaut, L. Y., \& Greenstein, J. S. (1986). Optimizing the touch tablet: The effects of control-display gain and method of cursor control. Human Factors, 28, 717-726.

Buck, L. (1980). Motor performance in relation to control-display gain and target width. Ergonomics, 23, 579-589.

Burr, D. C., \& Ross, J. (1982). Contrast sensitivity at high velocities. Vision Research, 22, 479-484.

Campbell, F., \& Maffei, L. (1981). The influence of spatial frequency and contrast on the perception of moving patterns. Vision Research, 21, 713-721.

Cavanagh, P. R., \& Komi, P. V. (1979). Electromechanical delay in human skeletal muscle under concentric and eccentric contractions. European Journal of Applied Physiology, 42, 159-163.

Council for International Organizations of Medical Sciences. (2002). International ethical guidelines for biomedical research involving human subjects. Retrieved from http:// www.cioms.ch/guidelines_nov_2002_blurb.htm

Craik, K. J. W. (1947). Theory of the human operator in control systems: I. The operator as an engineering system. British Journal of Psychology, 38, 56-61.

Craik, K. J. W. (1948). Theory of the human operator in control systems: II. Man as an element in a control system. British Journal of Psychology, 38, 142-148.

Elkind, J. I., \& Sprague, L. T. (1961). Transmission of information in simple manual control systems. IRE Transactions on Human Factors in Electronics, HFE-2, 58-60.

Elliot, D., Lyons, J., \& Dyson, K. (1997). Rescaling an acquired discrete aiming movement: Specific or general motor learning? Human Movement Science, 16, 81-96.

Engel, K. C., Anderson, J. H., \& Soechting, J. F. (2000). Similarity in the response of smooth pursuit and manual tracking to a change in the direction of target motion. Journal of Neurophysiology 84, 1149-1156.

Etschberger K. (1973). Leistungsfähigkeit und Regelungsverhalten des Menschen bei der Nachführung kontinuierlicher stochastischer Signale [Efficiency and control performance of man in 
following stochastic signals] (Dissertation). Technical University of Munich, Germany.

Etschberger K. (1975). Leistungsfähigkeit und Regelungsverhalten des Menschen bei der Nachführung stochastischer Signale [Efficiency and control performance of man in following stochastic signals]. Biological Cybernetics, 17, 81-98.

Ferrel, C., Leifflen, D., Orliaguet, J. P., \& Coello, Y. (2000). Pointing movement visually controlled through a video display: Adaptation to scale change. Ergonomics, 43, 461-473.

Fitts, P. M. (1954). The information capacity of the human motor system in controlling the amplitude of movement. Journal of Experimental Psychology, 47, 381-391.

Foulkes, A. J., \& Miall, R. C. (2000). Adaptation to visual feedback delays in a human manual tracking task. Experimental Brain Research, 131, 101-110.

Gottlieb, J. (2007). From thought to action: The partial cortex as a bridge between perception, action, and cognition. Neuron, 53, 9-16.

Holst, E., \& Mittelstaedt, H. (1950). Das Reafferenzprinzip (The reafference principle). Naturwissenschaften, 37, 464-476.

Jagacinski, R. (1977). A qualitative look at feedback control theory as a style of describing behaviour. Human Factors, 19, 331-347.

Jagacinski, R., \& Flach, J. (2003). Control theory for humans: Quantitative approaches to modeling performance. Mahwah, NJ: Lawrence Erlbaum.

Jenkins, W. L., \& Connor, M. B. (1949). Some design factors in making settings on a linear scale. Journal of Applied Psychology, 36, 269-271.

Joiner, W. M., \& Shelhamer, M. (2009). A model of time estimation and error feedback in predictive timing behaviour. Journal of Computational Neuroscience, 26, 119-138.

Khalid, A., Huey, J., Singhose, W., Lawrence, J., \& Frakes, D. (2006). Human operator performance testing using an inputshaped bridge crane. Journal of Dynamic Systems, Measurement, and Control, 128, 853-841.

Knight, J. L. (1987). Manual control and tracking. In G. Salvendy (Ed.), Handbook of human factors (pp. 182-218). New York, NY: Wiley.

Maioli, C., Falciati, L., \& Gianesini, T. (2007). Pursuit eye movements involve a covert motor plan for manual tracking. Journal of Neuroscience, 27, 7168-7173.

McRuer, D. T. (1980). Human dynamics in man-machine systems. Automatica, 16, 237-253.

McRuer, D. T. (1995). Pilot-induced oscillations and human dynamic behavior (NASA Contractor Report 4683). Washington, DC: NASA.

McRuer, D. T., Allen, R. W., Weir, D. H., \& Klein, R. H. (1977). New results in driver steering control models. Human Factors, 19, 381-397.

McRuer, D. T., Graham, D., Krendel, E. S., \& Reisener, W. (1965). Human pilot dynamics in compensatory systems: Theory models and experiments with controlled element and forcing function variations (AFFDL-TR-65-15). Wright-Patterson AFB, $\mathrm{OH}$ : Air Force Flight Dynamics Lab.

McRuer, D. T., \& Krendel, E. S. (1957). Dynamic response of human operators (WADC-TR-56-524). Wright-Patterson AFB, OH: Wright Air Development Center.

Miall, R. C. (1996). Task-dependent changes in visual feedback control: A frequency analysis of human manual tracking. Journal of Motor Behaviour, 28, 125-135.
Miall, R. C., Weir, D. J., \& Stein, J. F. (1985). Visuomotor tracking with delayed visual feedback. Neuroscience, 16, 511-520.

Miall, R. C., \& Wolpert, D. M. (1996). Forward models for physiological motor control. Neural Networks, 9, 1265-1279.

Paul, R. (2004). Elektrotechnik für Informatiker mit MATLAB und Multisim [Electrical Engineering for computer scientists using MATLAB and Multisim]. Stuttgart, Germany: Teubner Verlag.

Potter, J. J., \& Singhose, W. (2013). Improving manual tracking of systems with oscillatory dynamics. IEEE Transactions on Systems, Man, and Cybernetics-Part A: Systems and Humans, $43,46-52$.

Poulton, E. C. (1974). Tracking skill and manual control. London, UK: Academic Press.

Reed, D., Liu, X., \& Miall, R. C. (2003). On-line feedback control of human visually guided slow ramp tracking: Effects of spatial separation of visual cues. Neuroscience Letter, 338, 209-212.

Roerdink, M., Peper, C. E., \& Beek, P. J. (2005). Effects of correct and transformed visual feedback on rhythmic visuo-motor tracking: Tracking performance and visual search behaviour. Human Movement Science, 24, 379-402.

Rouse, W. B., \& Gopher, D. (1977). Estimation and control theory: Application to modeling behavior. Human Factors, 19, 315-329.

Sarlegna, F. R., Baud-Bovy, G., \& Danion, F. (2010). Delayed visual feedback affects both manual tracking and grip force control when transporting a handheld object. Journal of Neurophysiology, 104, 641-653.

Seidler, R. D., Bloomberg, J. J., \& Stelmach, G. E. (2001). Context-dependent arm pointing adaptation. Behavioural Brain Research, 119, 155-166.

Sheridan, T. B., \& Ferrell, W. R. (1974). Man-machine-systems. Cambridge, MA: MIT Press.

Stepp, N. (2009). Anticipation in feedback-delayed manual tracking of a chaotic oscillator. Experimental Brain Research, 198, 521-525.

Strasser, H., \& Platzer, H. (1972). Ein Meßplatz zur Bewertung psycho- bzw. sensumotorischer Fähigkeiten des Menschen [A test site to evaluate human psychological and sensorimotor skills]. Biomedizinische Technik, 17, 130-137.

Unbehauen, H. (2008). Regelungstechnik 1, Klassische Verfahren zur Analyse und Synthese linearer kontinuierlicher Regelsysteme, Fuzzy-Regelsysteme [Control Theory 1, conventional procedures to analyze and synthesize linear and continuous control systems, fuzzy control systems]. Braunschweig, Germany: Vieweg Friedrich+Sohn Verlag.

Van Doorn, R., \& Unema, P. (2005). Effects of adaptation to altered display gain on the control of single aimed movements. Motor Control, 9, 3-22.

Voss, H. U., McCandliss, B. D., Ghajar, J., \& Suh, M. (2007). A quantitative synchronization model for smooth pursuit target tracking. Biological Cybernetics, 96, 309-322.

Wickens, C. W. (1986). The effects of control dynamics on performance. In K. R. Boff, L. Kaufman, \& J. P. Thomas (Eds.), Handbook of perception and human performance: Vol. 2. Cognitive processes and performance (pp. 1-39). New York, NY: Wiley.

Wickens, C. W. (1992). Engineering psychology and human performance (2nd ed.). New York, NY: HarperCollins.

Wolpert, D. M., Miall, R. C., Winter, J. L., \& Stein, J. F. (1992). Evidence for an error deadzone in compensatory tracking. Journal of Motor Behaviour, 24, 299-308. 
Hans Gerisch received his diploma in aerospace engineering from the Bundeswehr University Munich, Germany, in 2001. Now he serves as a pilot in the German Air Force. Aside from that principal professional activity, he is working as a $\mathrm{PhD}$ candidate at the Institute of Communications Engineering of the Bundeswehr University Munich.

Gerhard Staude received his $\mathrm{PhD}$ and habilitation degrees from the Bundeswehr University Munich in 1996 and 2004, respectively. Currently he is an assistant professor in the Faculty of Electrical Engineering of the Bundeswehr University Munich.
Werner Wolf received his $\mathrm{PhD}$ degree from the Technical University Munich in 1978. He is a member of the Faculty of Electrical Engineering of the Bundeswehr University Munich and program officer of the IEEE German EMBS Chapter.

Gerhard Bauch received his $\mathrm{PhD}$ degree in electrical engineering from the Technical University Munich in 2001. Currently he is a full professor in the Institute of Communications Engineering of the Hamburg University of Technology and a senior member of the IEEE.

Date received: October 3, 2011

Date accepted: January 24, 2013 